\title{
Hybrid Spectrum Allocation Scheme in Wireless Cellular Networks
}

\author{
Rejoy George, Osianoh Glenn Aliu, Muhammad Ali Imran \\ India-UK Advanced Technology Centre of Excellence in Next Generation Networks \\ Centre for Communication Systems Research \\ University of Surrey, Guildford, United Kingdom \\ Email: rejoyg@gmail.com, \{0.aliu, m.imran\}@surrey.ac.uk
}

\begin{abstract}
Mobile services have seen a major upswing driven by the bandwidth hungry applications thus leading to higher data rate requirements on the wireless networks. Spectrum being the most precious resource in the wireless industry is of keen interest. Various spectrum assignment and frequency reuse schemes have been proposed in literature. However in future networks, dynamic schemes that adapt to spatio-temporal variation in the environment are desired. We thus present a hybrid spectrum assignment scheme which adapts its allocation strategies depending on user distribution in the system. Results show that the proposed dynamic spectrum assignment strategy improves spectrum utilization thereby providing a higher data rate for the users.
\end{abstract}

Keywords- LTE, Self-Organization, Dynamic Spectrum Access, Load balancing, Interference Coordination.

\section{INTRODUCTION}

The dynamic nature of mobile traffic makes it elusive and more tedious for the network designers to realize a perfect system. Manual optimization of the system would not yield the desired results. The operational costs involved in the manual optimization techniques tend to be on the higher side. The 3GPP for its LTE-A specifications has recommended a solution to have a self-organized cellular network, integrating the basic functionalities of self-configuration, self-optimization and selfhealing. Load balancing, to match the dynamics of the nonuniform user distribution has been identified as one of the use case for self-optimization functionality. The main motivation for this is the fact that over a period of time we can observe steady transition of traffic from one location to another. Achieving a fair resource scheduler is indeed a major requirement for self-optimization.

Another important use case identified is the interference coordination, which in turn affects the data rate that can be offered by the system. The data rate requirements of the new bandwidth hungry applications driven by the smart-phone industry has put a greater constraint on the wireless operators worldwide. The high data rate requirement is constrained by the spectrum availability. Spectrum is an extremely precious resource in the industry and also one which has remained heavily under-utilized because of the technological impairments and governmental regulations. An efficient spectrum management policy can alleviate the spectrum scarcity by intelligent channel assignment schemes. The radio resource management thus performed has the advantage of interference coordination as well, by considering the cochannel interference as a limiting factor while deciding the spectrum assignment strategy. If an intelligent system is indeed designed with the traffic density under each cell, it lends the ability for the system to adapt to the dynamics of traffic and thus deliver a more comprehensive solution.

We present a review on state of the art in self optimized load balancing schemes (Section II). Section III presents a mathematical framework. Due to the adaptive nature of the real systems, we demonstrate an algorithm for spectrum assignment which is based on neighbor cell list generated from automatic neighbor relation and load concentration awareness amongst the neighboring sectors.

\section{STATE OF THE ART}

\section{A. Self-Optimized Load Balancing}

Self-Organization is currently being investigated under the SOCRATES project which has identified twenty-five use cases that needs to be addressed by self-optimization functionalities [1]. The challenge in uniting all these together and build a single algorithm is the biggest challenge faced by the Telecom community [2]. The parameter optimization may lead to oscillatory and unstable behavior because of its closed nature and each of the parameter exerting influence on many of the goals. Thus a need for a coordination mechanism has been highlighted in [2] which have split the optimization parameters into different functional groups based on correlation.

Load balancing is one of the major use case identified and a factor with far-reaching industrial impact to provide a fair resource scheduling with limited resources. Many papers have focused on load balancing by optimizing the handoff threshold [3] and [4]. The algorithm followed in these papers was that of offloading the overloaded cells into its neighboring sectors by adjusting the handoff threshold in a coordinated manner so as to push the users lying near the boundary of the sectors to be associated to its less crowded neighbors. The interference level that may delimit the scheme has not been investigated in these papers. Also they have not demonstrated how this fixed/static threshold limit will prevent possible handoff ping pong effect between cells. 


\section{B. Interference Coordination}

Interference coordination is yet another important use case that may have impact on the offered throughput by the system. This has a direct effect on the QoS (quality of service) and lower satisfaction to users which are served with low SINR (Signal to Interference and Noise Ratio), usually the edge users. A self-organized Reuse Partitioning technique was pointed out in [5] that targeted the interference minimization between co-channel cells by an orthogonal channel allocation and thus providing enough spatial separation to bring down the co-channel interference to a minimum. Again the focus was only on interference minimization. No considerations were given for the traffic carried by each cell and a fair resource scheduling.

\section{SYSTEM MODEL}

A mathematical model has been formulated in [3], which defines a framework for simulations in the perspective of Self organized network (SON). The mathematical framework which characterizes a network layout and user position along with all the propagation characteristics explored in detail.

\section{A. Cellular Network}

- $\quad$ Each network node is placed at the position of $\vec{p}_{c}$ which defines a single base station serving three hexagonal sectors. Cloverleaf sectoring technique has been used for the layout as shown in Fig 1.

- Users being served by the cells are defined by the position $\vec{q}_{u}$, assumed to be randomly located over the entire coverage area. Since the investigations are mainly aimed at load balancing, a non-uniform distribution is assumed with particular areas overloaded purposefully to test the efficiency of the algorithm.

\section{B. Propagation Model}

A propagation model is generated which characterizes the wireless channel losses for the signal while being transmitted from the base station to the user. As explained in [6], the multiplicative noise endured by the signal can be further subdivided into distance dependant path loss, shadowing and fast fading. Since the SON algorithms are to be tracked over a period of time, the fast variations in the system may be neglected. Hence the fast fading component is neglected from the simulations.

- Distance dependant path loss is typically expressed as

$$
L_{P L}=l_{A}+l_{B} \cdot \log _{10}(d / K m)
$$

Where $d$ is the distance of each user from the base station given by $\left|\vec{p}_{c}-\vec{q}_{u}\right|$. Typical values for $l_{A}$ and $l_{B}$ are $148.1 \mathrm{~dB}$ and $37.6 \mathrm{~dB}$ [7].

- Shadowing is the characteristic feature in the propagation with which prevents line-of-sight (LOS) communication between the base station and user. This effect introduces non-linearity in an otherwise

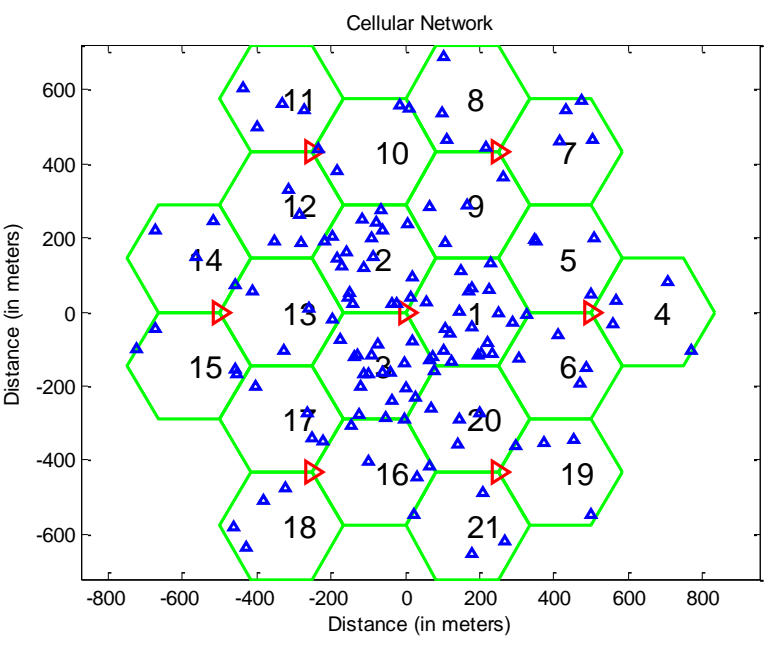

Fig 1: Cellular network layout for simulations

linear loss model thus replicating a practical scenario, and more realistic solutions. The shadowing maps are generated randomly from log-normal distribution with zero mean and standard deviation.

- The directional pattern of the antennas contributes to the propagation loss. The Radio Frequency (RF) antennas used at the base station radiates outwards with a pattern resembling a beacon shape. Cloverleaf sectoring has been assumed for the simulation with maximum antenna gain at the bore-sight angle. The azimuth and elevation pattern expressions are followed as given in [7] and [8].

- Thus the sum total of the three multiplicative components characterizes the propagation model.

$$
L_{c}\left(q_{u}\right)=L_{P L}+L_{M c}+L_{B}
$$

\section{Cell Association Function}

Each user is assumed to be equipped with an omnidirectional antenna having unity gain in all directions. During the downlink transmission mode, the signals from all the base station arrive at the user, with each signal undergoing uncertain levels of attenuation depending on the distance and shadowing effects. The signal strength at the user is measured for each user against all the base station antennas. The signal to interference and noise ratio (SINR) is thus computed assuming each sector as the primary serving sector and all the others as interference. The expression for SINR is given by

$$
S_{I N R}=\frac{P_{X(u), K(u)} * L_{c}\left(q_{u}\right)}{N+\sum_{c=S \backslash c, k=K(u)} P_{c} * L_{c}\left(q_{u}\right)}
$$

Where $P_{X(u), K(u)}, N$ and $L_{c}\left(q_{u}\right)$ are the transmit power of the base station antenna per frequency channel, AWGN noise and propagation loss perceived by each user respectively and $\mathscr{E}$ is the set of all sectors given $u$ users and $c$ cells. Now the association function can be expressed as

$$
X(u)=\max \left(\sum_{c} S I N R_{u}\right)
$$


From the set of SINR values for each user against all the base station antennas, the sector with highest SINR value can be assumed to be the primary serving sector for the user. Thus each user is now associated to one particular sector which acts as the serving sector.

\section{Load Concentration}

Once the users have been associated to a particular sector we can have an estimate of the percentage of users being served by each sector. The Load concentration $L(c)$ gives a picture of how loaded a sector is when compared to its neighbors. This is also the most essential term in performing radio resource management in a dynamic manner taking into account the present network requirements.

$$
L(c)=\frac{\sum_{c=X(u)} U}{\sum_{c} U}
$$

Where $L(c)$ represents the load concentration corresponding to each sector and $U$ represents the number of users present in each sector. The load concentration metric gives only the comparative figure the sector is carrying with the whole system. This does not lend the adaptive nature needed for tackling load balancing.

\section{RESULTS}

In order to have a comparison, we need to first build a benchmark against which the SINR and data rate values can be compared to measure the overall improvement. The two different benchmarks that will be used for simulation includes full frequency reuse and static channel allocation scheme.

\section{A. Full Frequency Reuse and Static Channel Allocation}

In full frequency reuse scheme, the entire spectrum is available for transmission across all the sectors. The main advantage of this is high spectrum utilization and ability to handle maximum number of users. But the interference level with this scheme is very high, particularly for users lying at the edge of the sectors. In order to reduce the interference levels, we can introduce an orthogonal approach for channel allocation

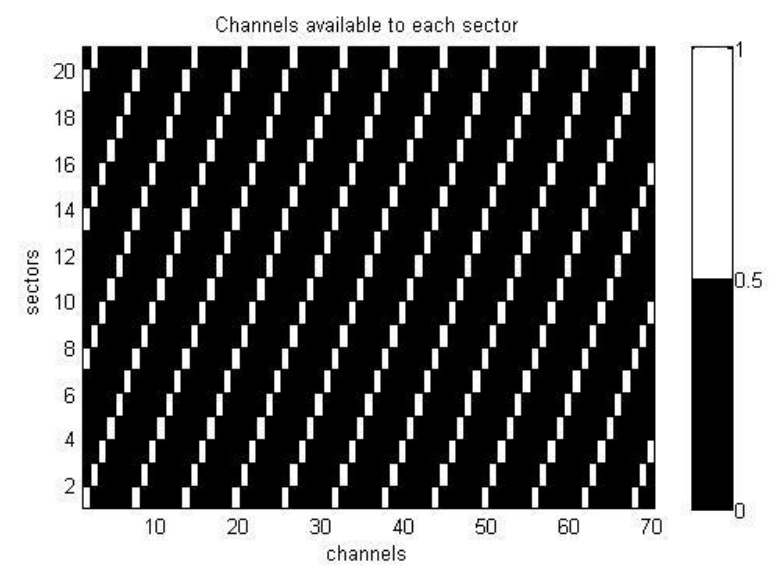

Fig 2: Static Channel Allocation pool in such a manner that no neighbors share the same channel for transmission as shown in Fig 2. This orthogonal approach can indeed help achieve significant reduction in interference levels. However, since only a portion of the spectrum is being utilized in each sector, this leads to low spectrum utilization, thus bringing down the overall data rate in the system. This static channel allocation is a predetermined resource allocation technique, which does not provide any adaptability to the network requirements. Hence static allocation is certainly not preferred as an alternative because of its low spectrum utilization and inability to handle non-uniform traffic. But the SINR levels achieved by this method forms a benchmark to be attained.

\section{PROPOSED AlLOCATION SCHEMES}

\section{A. Automatic Neighbour Relation}

The adaptive nature of the algorithm is determined by its ability to sense the state of its surrounding neighbors. The awareness again depends on how we classify the individual sectors as interfering neighbors. The neighbor relation can be notated as follows.

$$
\mathcal{N}=\left[n_{i, j} / n_{i, j} \in\{0,1\}\right]_{S X S}
$$

Where $\mathcal{N}$ is $S X S$ square matrix depicting the interfering neighbors.

$n_{i, j}=\left\{\begin{array}{c}1, \text { sector } i \text { and } j \text { interfere witheach other and } i \neq j \\ 0, \text { otherwise }\end{array}\right.$

The identification of neighbors forms the most basic step towards building a learning algorithm which has the capability to adapt to the load mobility.

\section{B. Dynamic Channel Allocation}

The adaptive nature of the system can be incorporated by two factors, automatic neighbor relation and load concentration awareness amongst the neighboring sectors. Here we introduce an algorithm which allocates channels based on the above mentioned factors.

Once we have an allocation pool for each sector determined dynamically based on the load concentration in the adjacent neighbors, the base station transmits signal to the user from one of the channels available to the particular sector. Since the cochannels cells are now spatially separated by a good distance, the interference levels will come down and be comparable to

\begin{tabular}{|c|c|}
\multicolumn{2}{|c}{ Table 1: Set notations used } \\
\hline Set & Definition \\
\hline $\mathcal{M}$ & Set of all sectors in the network \\
\hline $\mathcal{N}$ & Set of all channels \\
\hline $\mathcal{C}$ & Conflicting set of channels \\
\hline $\mathcal{O}$ & Set of available channels \\
\hline $\mathcal{H}$ & Set of channels allocated to each sector \\
\hline
\end{tabular}




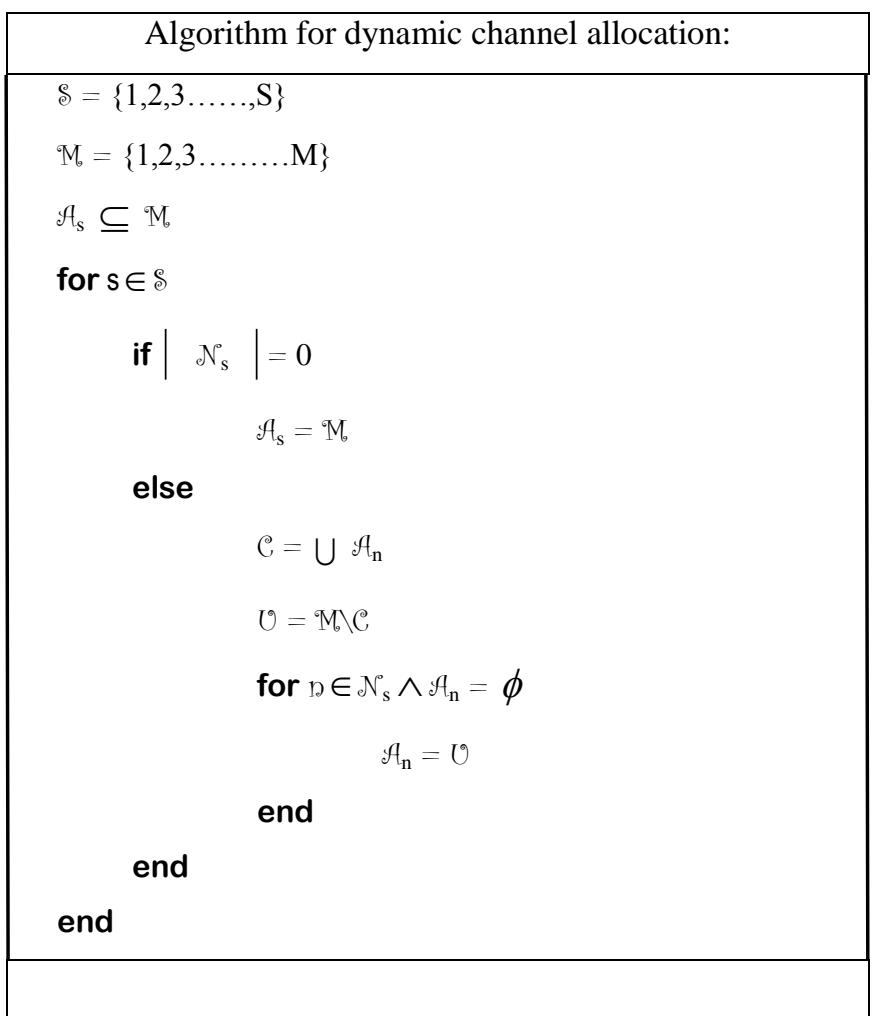

the static allocation technique with one major advantage over it, being able to handle non-uniform user distribution. As shown in Fig 3, the orthogonality among neighboring sectors is maintained and the spectrum utilization factor has also increased considerably.

The data rate can be computed using the following equation

$$
C=B W^{*} \gamma * \log _{2}(1+\operatorname{SINR})
$$

Where $B W$ and $\gamma$ is the channel bandwidth and channel utilization factor respectively (highlighted in Fig 5). The improvement in the channel utilization factor directly influences the data rate values which is now considerably higher than the static allocation technique.

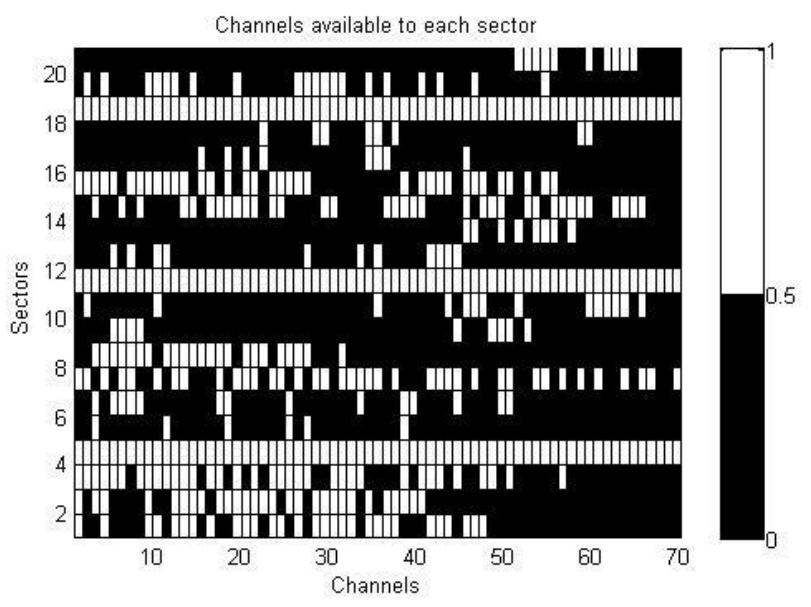

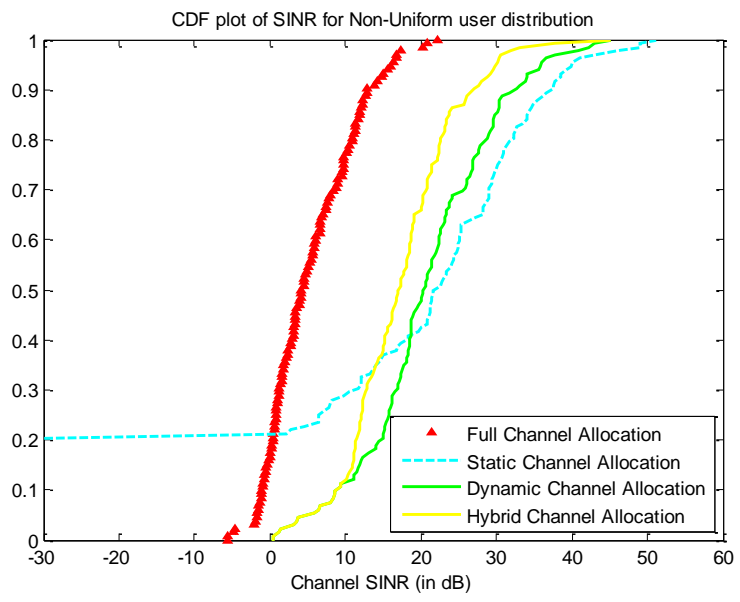

Fig 4: SINR comparison between the four channel assignment techniques

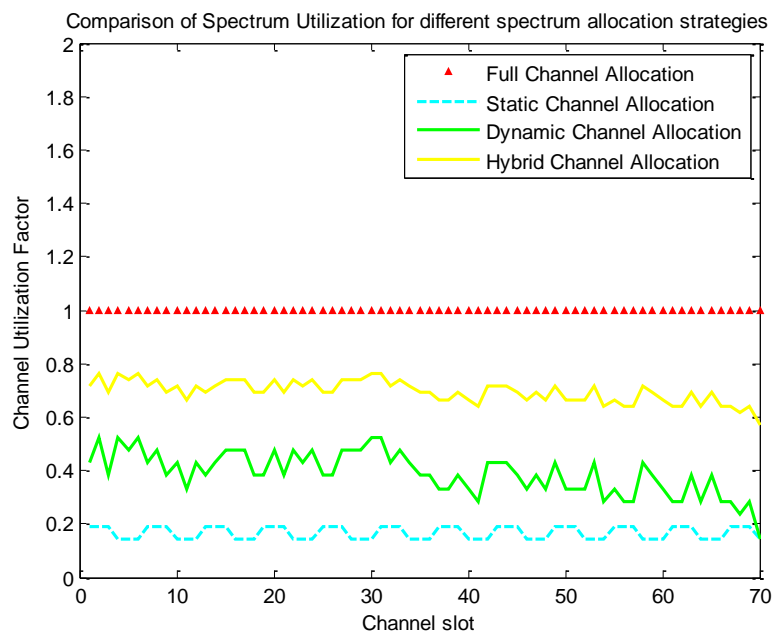

Fig 5: Channel Utilization factor

\section{Hybrid Channel Allocation}

The scope for improvement in terms of spectrum utilization is still high. The most important factor that can prove decisive in this regard is the spatial separation between the users. The users located close to the cell centre are less likely to be influenced by interference from the neighboring sectors, since the SINR for these users are indeed quite high. Thus, by reusing all the frequency channels in all the sectors in such a manner that the users being served by high SINR margin chooses channels from the full frequency reuse pool for transmission. Whereas the users at the edge of the sectors, which are termed as critical users can be allocated channels from the dynamic spectrum pool which takes into consideration the adjacent sector channel allocation, thus providing enough spatial separation between co-channel users to improve the overall SINR. The tradeoff with this hybrid allocation scheme is that the performance of non-critical users is sacrificed by a small margin to ensure fairness.

Fig 3: Dynamic channel allocation pool 


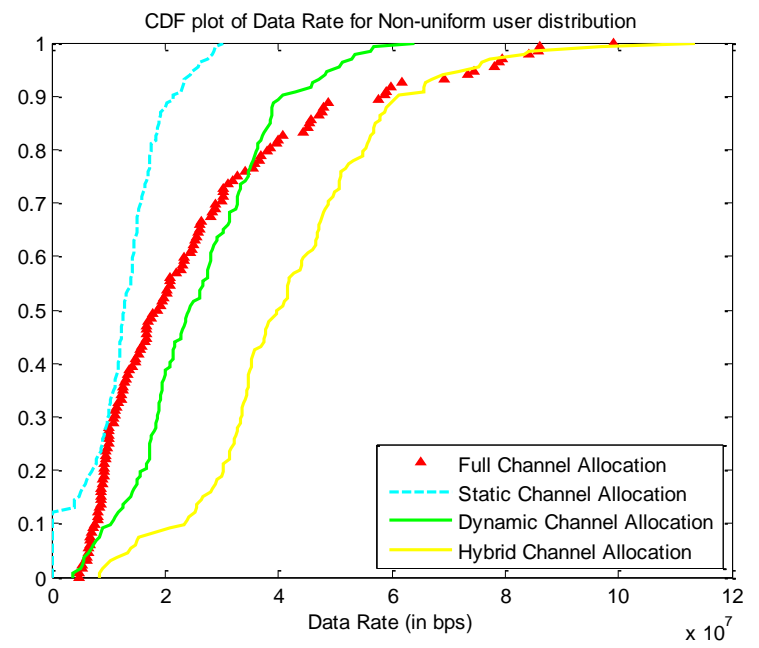

Fig 6: Data Rate comparison between the different channel allocation strategies

This can be considered a good tradeoff since interference degradation observed due to this technique is by far very less and still serves with much better signal strength as compared to full frequency reuse technique which is evident from Fig 4.

On the other hand the positive aspects include better signal strength for critical users $\left(\mathrm{SINR}_{\mathrm{u}}<10 \mathrm{~dB}\right)$, ability to handle non-uniform user distribution effectively, higher spectrum utilization as against dynamic frequency allocation and most importantly the better overall data rate offered highlighted in Fig 5 and Fig 6 respectively.

\section{Evaluation of performance in a sparse system model}

The simulations were performed considering dense system with an inter-site distance of 500 meters, which replicates an urban model. For result validation, a rural model characterized by an inter-site distance of 3000 meters is now used for performing simulations. All the other parameters conform to [7]. The resulting data rate plot in Fig 7 shows a merging of the data rate for the hybrid allocation strategy and full frequency reuse, mainly attributed to the distance between the base station antennas which being far away tends to have less influence on the neighboring sectors. Thus for a cellular system with high inter-site distance, the use of full frequency reuse is more effective which provides a high spectrum utilization factor as well.

\section{CONCLUSION}

In this paper, we have demonstrated a spectrum assignment strategy in a cellular system which autonomously adapts the variations in traffic and allocates sub bands of spectrum based on the network requirements in order to minimize inter-cell interference thus improving system performance and also maintains high spectrum utilization. We have highlighted how the full frequency reuse technique is helpful in the case of sparse system model and how the technique fails to achieve similar efficiency in a dense model.

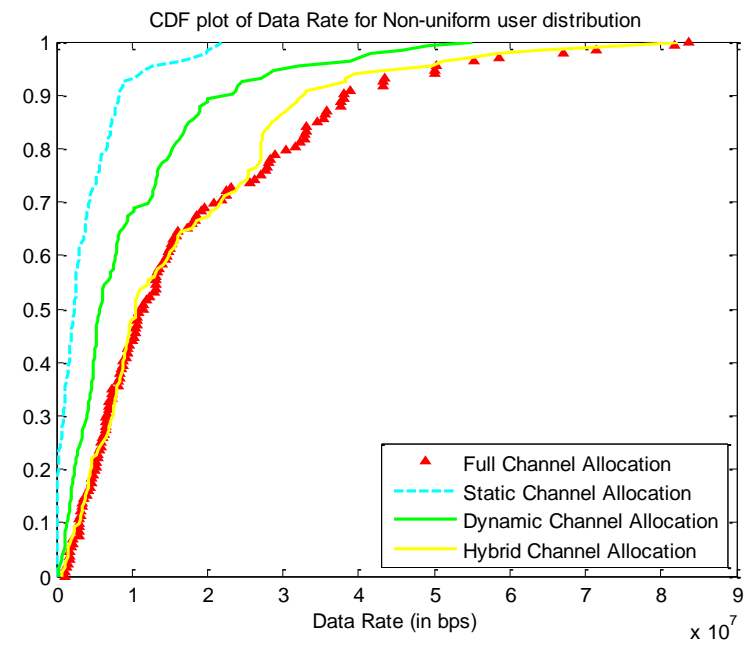

Fig 7: Data rate comparison between different channel allocation schemes in sparse system model

We thus proposed a self optimizing spectrum assignment strategy which should switch between with a full frequency reuse for sparse (rural) systems and a hybrid allocation scheme in a dense (urban) system. This can achieve significant improvement in network quality as compared to implementing a single spectrum assignment scheme. The results shows better resource utilization and ideas for intelligent and autonomous implementation of this scheme is ongoing work.

\section{ACKNOWLEDGMENT}

The project is being funded by IU-ATC as a part of UKEPSRC Digital Economy Programme and the Government of India, Department of Science and Technology (DST).

\section{REFERENCES}

[1] M Amirijoo, et al, Use Cases, Requirements and Assessment criteria for future Self-organizaing radio access networks, in Self-Organizing Systems, Proceedings Vol 5343, K A Hummel and J P G Sterbenz, Eds, ed Berlin: Springer-Verlag Berlin,2008, pp. 275-280.

[2] T. Jansen, et al., Embedding Multiple Self-Organisation Functionalities in Future Radio Access Networks, 2009 IEEE Vehicular Technology Conference, 2009.

[3] Ingo Viering, Andreas Lobinger and Martin Doettling, A Mathematical perspective of self optimizing wireless networks, Nomor Research $\mathrm{GmbH}$, Munich, Germany, IEEE ICC 2009 proceedings.

[4] Andreas Lobinger et al, Load balancing in downlink LTE Self-optimizing Networks, Report EURO-COST, Athens, 2010.

[5] H. Furukawa and Y. Akaiwa, Self-organized reuse partitioning: A distributed dynamic channel assignment method in cellular systems, Electronics and Communications in Japan Part I-Communications, vol. 80, pp. 67-75, May 1997.

[6] Simon R Saunders, Alejandro Aragon Zavala, Antennas and Propagation for Wireless Communication Systems, Second Edition, John Wiley and Sons Inc, West Sussex, 2007.

[7] 3GPP TR 25.814 V7.1.0 (2006-09), Physical Layer aspects for evolved Universal Terrestrial Radio Access (UTRA) (Release 7), 3GPP support office, France, 2006.

[8] R. Hoppe, Comparison and Evaluation of algorithms for the interpolation of $3 D$ antenna patterns based on $2 D$ horizontal and $2 D$ vertical patterns, Study AWE Communications GmbH, V 1.0, 2003. 